\title{
Thermomechanical Coupling Damage and Analysis of Buckling Load of Laser Irradiated CFRP Cylinder Shell under Axial Load
}

\author{
Xiaodong XING, Weilong GUO, Xiaoming DUAN, Liquan WANG \\ College of Mechanical and Electrical Engineering, Harbin Engineering University, Harbin 150001, China, \\ E-mail:xingxiaodong@hrbeu.edu.cn

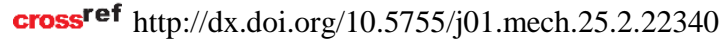

\section{Introduction}

CFRP, as a complex composite material, is commonly adopted in fabrication of these components in order to achieve lightweight and versatile structure [1-4]. The cylindrical shell is a typical load-bearing structure for aircraft components. When the CFRP cylindrical shell surface is exposed to a laser pulse, the irradiated area will experience a sudden temperature rise, which leads to stress concentration. As a result, the structure may be damaged to some extend if it is subjected to axial compression simultaneously. In this case, the thermodynamic parameters of materials are considered as temperature dependent [5-7]. Jianheng Zhao et al. presented the temperature distribution of the cylindrical shell irradiated by an intense laser pulse using FEM simulation [8]. Ji Wang et al. studied the thermodynamic responses and thermal buckling failure behavior of a pre-loaded cylindrical shell subjected to laser irradiation by numerical methods [9]. The buckling modes and eigenvalues of cylindrical shells subjected to axial compression or internal pressure were obtained, and the influences of laser intensity, irradiated duration, preloading conditions and the shell's geometric scale and shape on the thermal buckling failure modes were discussed as well. Deng et al. developed a finite element model for cylindrical thin shells subjected to axial compression under laser pulse irradiation, employing temperature dependent material parameters, and conducted finite element numerical analysis of thermal buckling failure $[10,11]$. Tafreshi et al. used the nonlinear FEM to study the response of composite cylindrical shells subject to combined loading [12]. Frulloni et al. presented the finite element modelling of the failure behaviour of lattice composite hollow structures that have been subjected to an external hydrostatic pressure [13]. However, the mechanism of thermomechanical coupling damage process of CFRP caused by laser irradiation is very complicated, so there is very limited research works reported. Further investigations need to be done for the cases like the effect of laser irradiation or thermomechanical coupling loading on the bearing capacity of CFRP structure, influence of different parameters, etc.

In this paper, the dynamic buckling failure process of laser irradiated CFRP cylindrical shell under axial compression was simulated by means of finite element quasistatic analysis method using ABAQUS, and the influence of the thermomechanical coupling damage on the buckling failure under different parameters was obtained.

\section{FEM model of laser irradiated CFRP cylinder shell under axial compression}

2.1. Heat transfer

The material of the cylindrical shell is T300/914 carbon fiber epoxy composite, the material has good heat resistance and low thermal conductivity [14]. When a body is exposed to a laser irradiation, the heat transfer process can be described by the Eq. (1) [14] on condition that the maximum temperature of the irradiated area is below the ablative temperature:

$$
\rho c \frac{\partial T}{\partial t}=\frac{\partial}{\partial x_{i}}\left(\lambda_{i j} \frac{\partial T}{\partial x_{j}}\right) \delta_{i j}-T \beta^{i j} \dot{\gamma}_{i j}+\dot{Q}_{l a s e r},
$$

where: $T(x, y, z, t)$ is temperature field at $t$ moment; $\rho, c$ and $\lambda$ are density, specific heat and thermal conductivity coefficient of the material respectively, which are correlation with the temperature and other factors; The $T \beta^{i j} \dot{\gamma}_{i j}$ term is the amount of heat caused by the deformation work; $\beta^{i j}$ is material property coefficient; $\dot{\gamma}_{i j}=\frac{1}{2}\left(\dot{u}_{i, j}+\dot{u}_{j, i}\right), \dot{u}_{i}$ is the change rate of displacement, this term represents the influence of the stress field (displacement field) on the temperature field, called coupling term; $\dot{Q}_{\text {laser }}$ is the laser heat source [14], which may be expressed as:

$$
\dot{Q}_{\text {laser }}=(1-R) \alpha_{a} q_{\text {inc }} e^{-\alpha_{a} z},
$$

and the laser heat source can be treated as body heat source or surface heat source, depending on the depth of absorption region of radiation. The laser heat source is dealt with as a boundary condition.

To simplify the practical situation, only conduction is taken into account, regardless of delivery of the energy through the cylindrical shell surface by radiation heat transfer. When the radiation equilibrium is established on the boundary, the overall heat flow yields,

$$
\begin{aligned}
& q_{\text {laser }}=q_{\text {cond }}, \\
& q_{\text {cond }}=\lambda_{n n} \frac{\partial T}{\partial n},
\end{aligned}
$$

where: $q_{\text {laser }}$ is the laser heat flow of surface absorption, is $q_{\text {cond }}$ the inside conduction heat flow of body, $n$ is the outer normal unit vector on the boundary, $\lambda_{n n}$ is the thermal conductivity in normal direction. 


\subsection{Laser heat source}

The spatial distribution of power density of continuous laser is considering as Gauss function:

$$
q_{\text {inc }}(r)=q_{\max } \exp \left(-2 r^{2} / R^{2}\right),
$$

where: $R$ is the radius of laser spot; $q_{\max }$ is maximum power density of laser irradiation, with $q_{\max }=2 q_{0} /\left(1-e^{-2}\right), q_{0}$ is average power density. The change of laser absorption coefficient is not taken into account in the calculation.

\subsection{Material parameters}

The thermal and mechanical properties of T300/914 carbon fibre epoxy composite are strongly temperature-dependent, and the values of different parameters at different temperatures were given in Table 1 and Table 2.

Table 1

Thermal property parameters of T300/914 carbon fibre epoxy composite

\begin{tabular}{|c|c|c|c|c|c|}
\hline Temperature $(\mathrm{K})$ & $\lambda_{11}(\mathrm{~W} /(\mathrm{m} \cdot \mathrm{K}))$ & $\lambda_{22}(\mathrm{~W} /(\mathrm{m} \cdot \mathrm{K}))$ & $\lambda_{33}(\mathrm{~W} /(\mathrm{m} \cdot \mathrm{K}))$ & $C(\mathrm{~J} / \mathrm{Kg} \cdot \mathrm{K})$ & $\rho\left(\mathrm{kg} / \mathrm{m}^{3}\right)$ \\
\hline 311 & 0.033 & 0.033 & 0.35 & 1027.778 & 1458 \\
\hline 350 & 0.034 & 0.034 & 0.37 & 1027.778 & 1458 \\
\hline 400 & 0.037 & 0.037 & 0.39 & 1027.778 & 1458 \\
\hline 561 & 0.044 & 0.044 & 0.47 & 1027.176 & 1455.95 \\
\hline 634 & 0.049 & 0.049 & 0.55 & 1018.824 & 1428.21 \\
\hline 700 & 0.056 & 0.056 & 0.80 & 981.7158 & 1316.74 \\
\hline 748 & 0.058 & 0.058 & 1.05 & 938.7894 & 1208.03 \\
\hline
\end{tabular}

Table 2

Mechanical properties of T300/914 carbon fibre epoxy composite

\begin{tabular}{|c|c|c|c|c|c|c|c|c|c|}
\hline Temperature $(\mathrm{K})$ & $E_{1}(\mathrm{~Pa})$ & $E_{2}(\mathrm{~Pa})$ & $E_{3}(\mathrm{~Pa})$ & $\mu_{12}$ & $\mu_{13}$ & $\mu_{23}$ & $G_{12}(\mathrm{~Pa})$ & $G_{13}(\mathrm{~Pa})$ & $G_{23}(\mathrm{~Pa})$ \\
\hline 311 & $1.87 \times 10^{9}$ & $1.79 \times 10^{9}$ & $1.79 \times 10^{9}$ & 0.3 & 0.31 & 0.31 & $4.72 \times 10^{8}$ & $4.72 \times 10^{8}$ & $4.72 \times 10^{8}$ \\
\hline 350 & $4.17 \times 10^{8}$ & $3.85 \times 10^{8}$ & $3.85 \times 10^{8}$ & 0.3 & 0.31 & 0.31 & $1.27 \times 10^{8}$ & $1.27 \times 10^{8}$ & $1.27 \times 10^{8}$ \\
\hline 400 & $3.90 \times 10^{8}$ & $3.61 \times 10^{8}$ & $3.61 \times 10^{8}$ & 0.3 & 0.31 & 0.31 & $1.20 \times 10^{8}$ & $1.20 \times 10^{8}$ & $1.20 \times 10^{8}$ \\
\hline 561 & $3.01 \times 10^{8}$ & $2.78 \times 10^{8}$ & $2.78 \times 10^{8}$ & 0.3 & 0.31 & 0.31 & $9.40 \times 10^{7}$ & $9.40 \times 10^{7}$ & $9.40 \times 10^{7}$ \\
\hline 634 & $2.59 \times 10^{8}$ & $2.39 \times 10^{8}$ & $2.39 \times 10^{8}$ & 0.3 & 0.31 & 0.31 & $8.16 \times 10^{7}$ & $8.16 \times 10^{7}$ & $8.16 \times 10^{7}$ \\
\hline 700 & $2.22 \times 10^{8}$ & $2.05 \times 10^{8}$ & $2.05 \times 10^{8}$ & 0.3 & 0.31 & 0.31 & $7.08 \times 10^{7}$ & $7.08 \times 10^{7}$ & $7.08 \times 10^{7}$ \\
\hline 748 & $1.97 \times 10^{8}$ & $1.82 \times 10^{8}$ & $1.82 \times 10^{8}$ & 0.3 & 0.31 & 0.31 & $6.33 \times 10^{7}$ & $6.33 \times 10^{7}$ & $6.33 \times 10^{7}$ \\
\hline 870 & $1.45 \times 10^{8}$ & $1.34 \times 10^{8}$ & $1.34 \times 10^{8}$ & 0.3 & 0.31 & 0.31 & $4.68 \times 10^{7}$ & $4.68 \times 10^{7}$ & $4.68 \times 10^{7}$ \\
\hline
\end{tabular}

\subsection{Finite Element Model}

The buckling failure of laser irradiated cylindrical shell under axial compression is thermomechanical coupling process, which involves heat transfer, ablation, mechanical load, and the geometric and material nonlinearities have to be dealt with. In this paper, the finite element model of a cylindrical shell with the dimensions of $L($ length $)=1 \mathrm{~m}$, $D$ (diameter $)=0.4 \mathrm{~m}, t$ (thickness $)=0.02 \mathrm{~m}$, was established with C3D8T element. The laser power is $8 \times 10^{5} \mathrm{~W} / \mathrm{m}^{2}$. One end of the cylinder was fixed, and the other end was subjected to a compressive displacement. A continuous laser irradiation was applied simultaneously until the cylinder underwent buckling failure. The entire thermomechanical coupling dynamic progress was simulated using ABAQUS.

\subsection{Calculation method}

Thermomechanical coupling damage effect on cylindrical shells is mainly due to the rapid increase of temperature in the irradiated region, which leads to a significant reduction in the bearing capacity of the cylindrical shell [15]. Thermomechanical coupling damage process of the CFRP cylindrical shell under axial compression and laser irradiation is very complicated, so simplification of the temperature dependent material properties has to be made in order to carry out the analysis. On this basis, the effect of thermomechanical coupling damage on CBL was analyzed, and the influence of some related parameters (including the thickness of cylindrical shell, the size of the spot, spot position) on the CBL was investigated.
The finite element analysis procedure was shown in Fig. 1. By the secondary development of ABAQUS, a Dflux subroutine was developed to apply a Gaussian heat flux to the shell surface. Then, temperature field of the CFRP cylindrical shell under laser irradiation was calculated, and the temperature-dependent material property parameters were determined once the node temperatures are obtained. Finally, the thermoelastic analysis was carried out. This finite element analysis strategy was validated by experiments in [16].

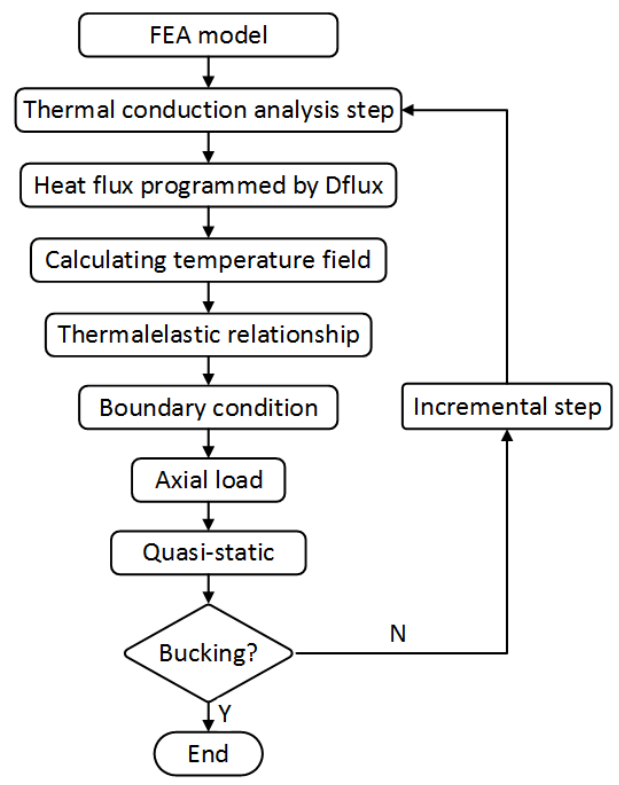

Fig. 1 Flowchart of numerical simulation 


\section{Results and discussion}

\subsection{Buckling failure}

Variation of the material mechanical parameters caused by laser irradiation is the main determining factor for the calculation of CBL. The material nonlinearity, geometric nonlinearity and boundary nonlinearity were also considered as well. Then, the quasi-static process of buckling failure of CFRP cylindrical shell under axial compression and laser irradiation was simulated by numerical calculation. The temperature field and the buckling mode were shown in Fig. 2. The compressive load applied to the cylinder end was shown in Fig. 3.
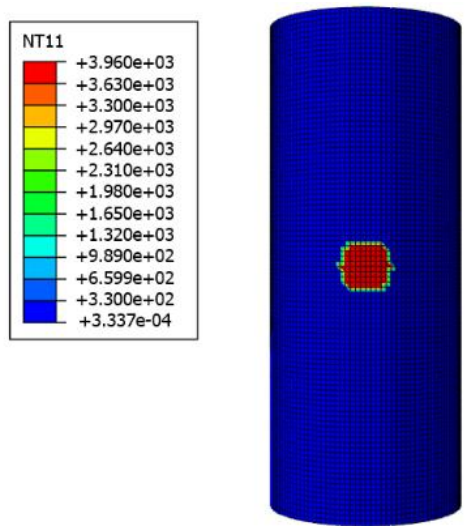

a
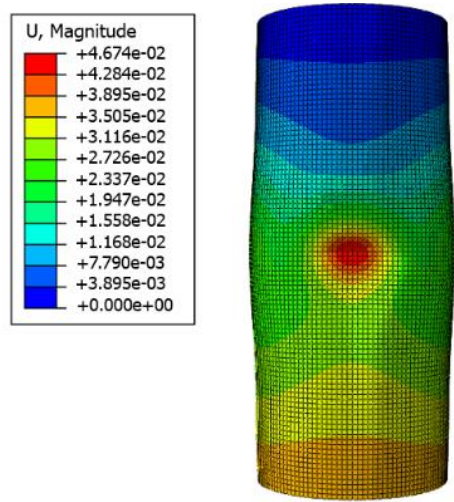

b

Fig. 2 The buckling failure of the CFRP cylindrical shell (a) Temperature field of the cylinder shell, (b) Buckling mode of the cylinder shell

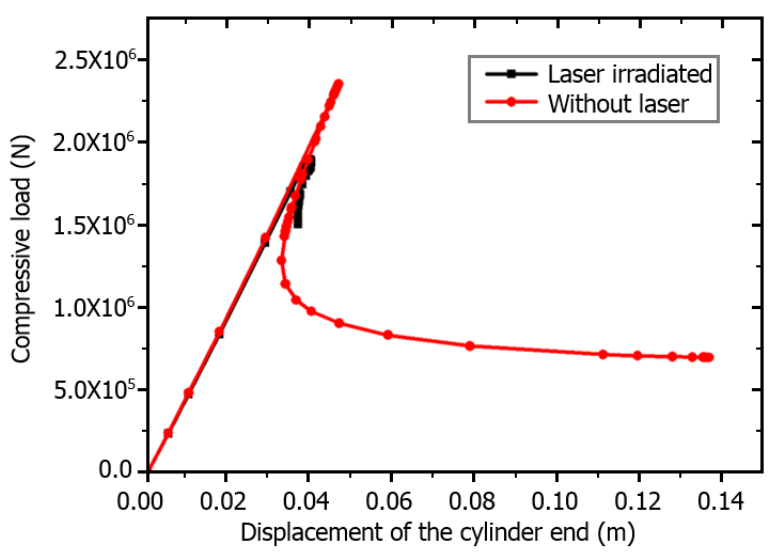

Fig. 3 The compressive load varied with the compressive displacement
Figs. 1 and 2 indicate that the laser irradiation has a great influence on the buckling threshold of the cylindrical shell. The laser irradiation can greatly decrease the value of CBL. Moreover, the global buckling occurs when there is no laser irradiation, and local buckling occurs due to laser irradiation.

\subsection{Effect of shell thickness on the CBL}

The thickness of the shell has an obvious influence on the CBL. In this case, the shell thickness was taken as $0.005 \mathrm{~m}, 0.01 \mathrm{~m}, 0.015 \mathrm{~m}, 0.02 \mathrm{~m}, 0.025 \mathrm{~m}$, the radius of the spot is $5 \mathrm{~mm}$, and the laser power density is $8 \times 10^{5} \mathrm{~W} / \mathrm{m}^{2}$. The CBLs at different cylindrical shell thickness was obtained by numerical calculation as shown in Fig. 4. According to Fig. 4, we can see that the buckling loads vary as quadratic function with the shell thickness, the thinner the cylindrical shell, the easier the buckling. It also appears that the buckling load decreases due to laser irradiation, and the thicker the shell thickness, the greater the effect. That can be attributed to the load capacity related to shell thickness strongly, so does the effect of the laser irradiation.

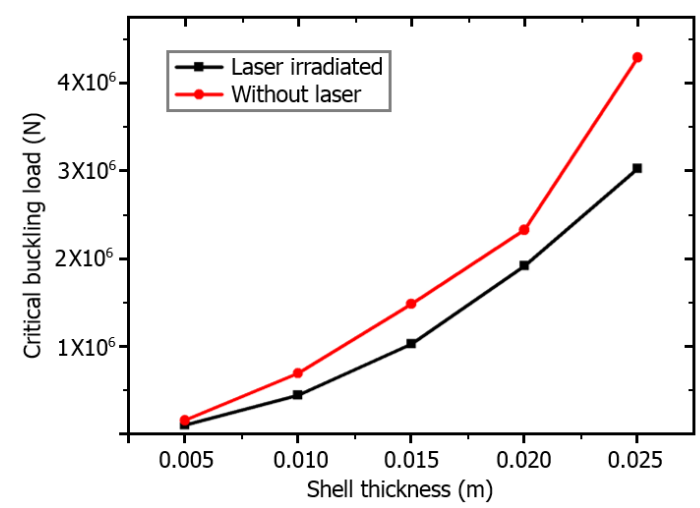

a

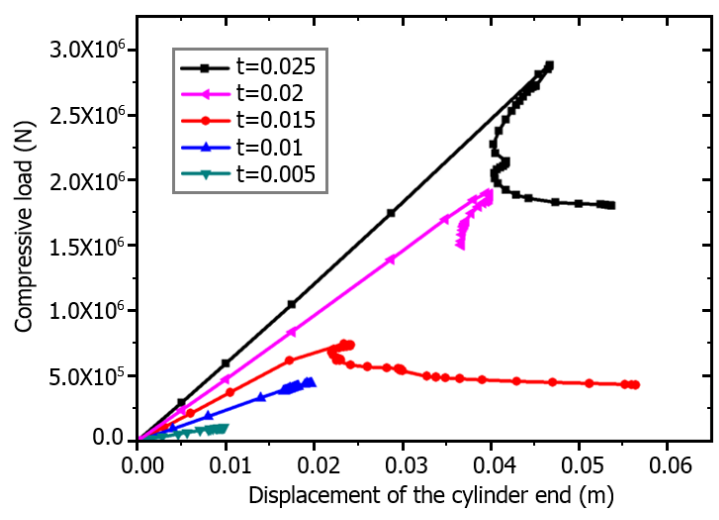

b

Fig. 4 The effect of the shell thickness on the CBL (a) The CBL at different shell thickness, (b) The compressive load varied with the compressive displacement

\subsection{Effect of the laser spot size on the CBL}

The effect of radius of the spot on the CBL is further studied. In this case, the thickness of the cylindrical shell is $0.02 \mathrm{~m}$, the laser power density is $8 \times 10^{5} \mathrm{~W} / \mathrm{m}^{2}$, and the radius of the spot is $0.01 \mathrm{~m}, 0.03 \mathrm{~m}, 0.04 \mathrm{~m}, 0.05 \mathrm{~m}, 0.07$ $\mathrm{m}, 0.1 \mathrm{~m}$. The relationship between the CBL and the radius of the spot was shown in Fig. 5. Fig. 5 indicates that the CBL 
of the cylindrical shell under laser irradiation and axial compression nonlinearly decreases with the increase of the ra-

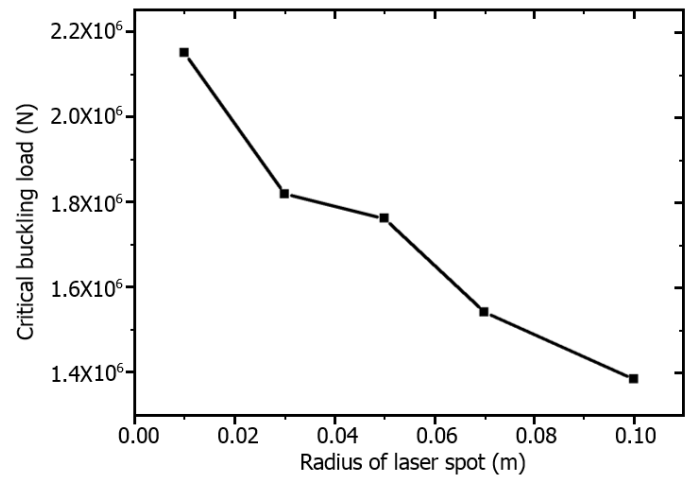

a dius of the spot. This is because more heat energy is absorbed by the material with the increase of the spot size.

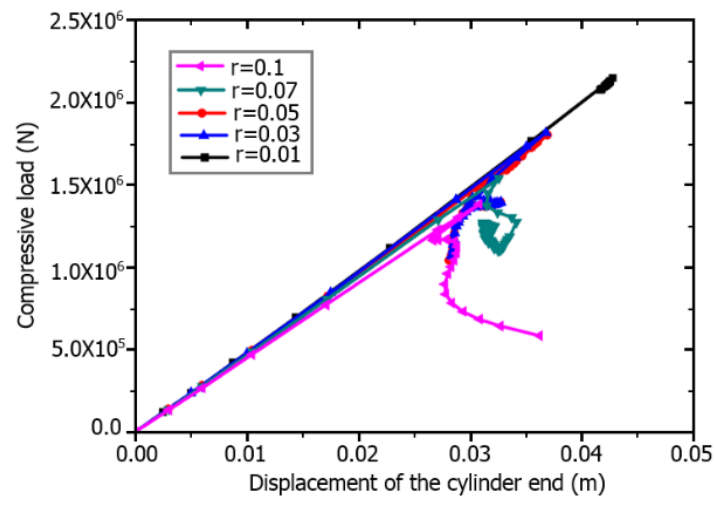

b

Fig. 5 The effect of the laser spot size on the CBL (a) The CBL varied with the laser spot size, (b) The compressive load varied with the compressive displacement

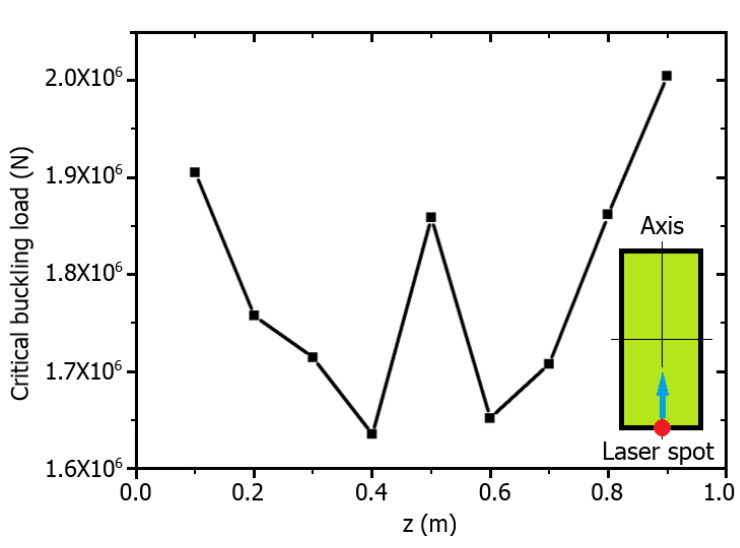

a

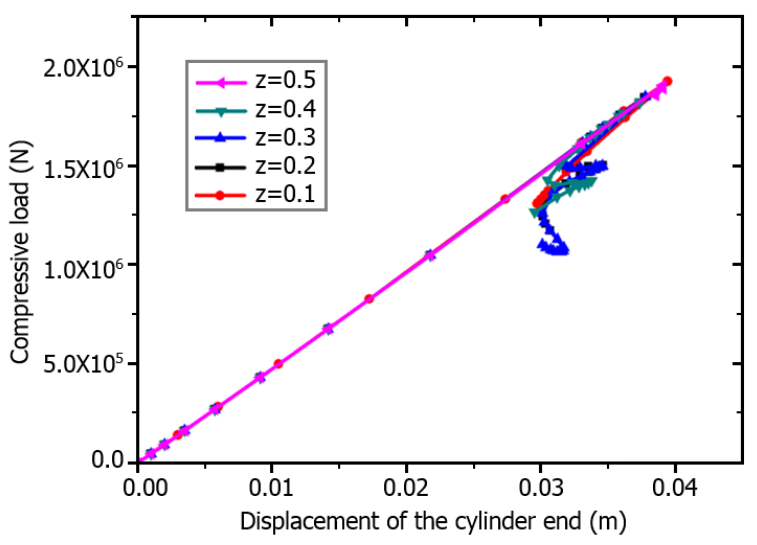

b

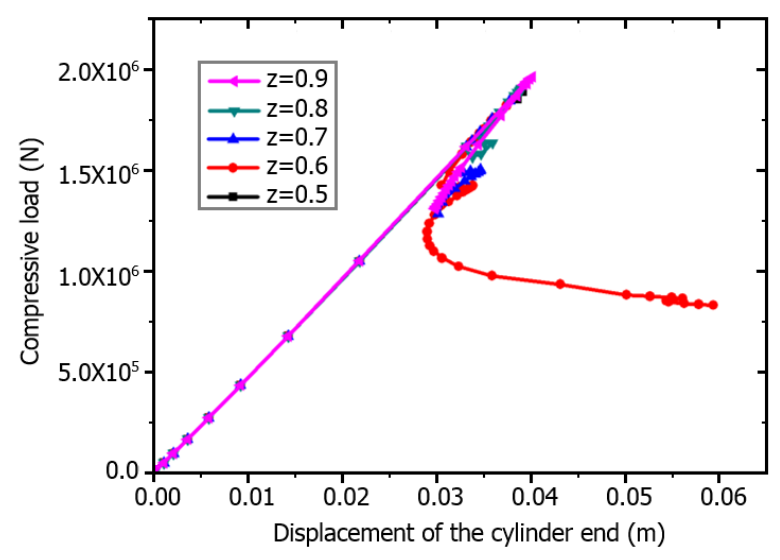

$\mathrm{c}$

Fig. 6 The effect of the laser spot position along the axis on the CBL (a) The CBL varied with different laser spot positions, (b) The compressive load varied with the compressive displacement with $z=0.1$ to $0.5 \mathrm{~m}$, (c) The compressive load varied with the compressive displacement with $z=0.5$ to $0.9 \mathrm{~m}$

\subsection{Effect of the laser irradiated position on the $\mathrm{CBL}$}

In order to find the location prone to damage in the cylindrical shell, the two following cases are discussed. The laser spot moves along the axis or deviates from the axis. In the first case, we take $z=0.1 \mathrm{~m}, 0.2 \mathrm{~m}, 0.3 \mathrm{~m}, 0.4 \mathrm{~m}, 0.5 \mathrm{~m}$, $0.6 \mathrm{~m}, 0.7 \mathrm{~m}, 0.8 \mathrm{~m}, 0.9 \mathrm{~m}$. The relationship between the spot position and the CBL was shown in Fig. 6, which indicates the CBL distribution is approximately symmetric about the middle section of the cylinder. The vulnerable position is not the midpoint on the axis, but two points approaching to the midpoint from two ends.

In the second case, the laser spot moves off the axis perpendicularly, and we take the distance $L=0 \mathrm{~m}, 0.05 \mathrm{~m}$, $0.1 \mathrm{~m}, 0.15 \mathrm{~m}$. The CBL with respect to the distance $L$ was obtained as shown in Fig. 7, and we can see that the CBL decreases with the increase of the deviating distance. In other words, the buckling tends to happen when the laser 
spot is far away from the axis.

Combining two cases, it can be concluded that the vulnerable location for the laser irradiation leading to buckling damage is not the geometrical centre, and it is due to the characteristic of buckling damage.

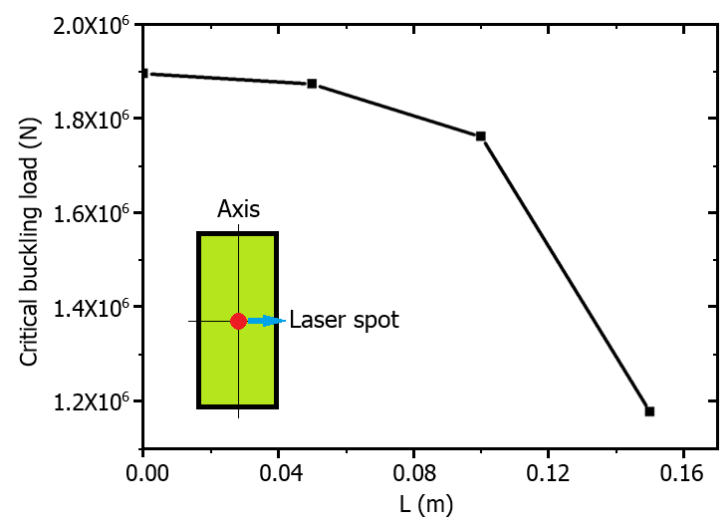

a

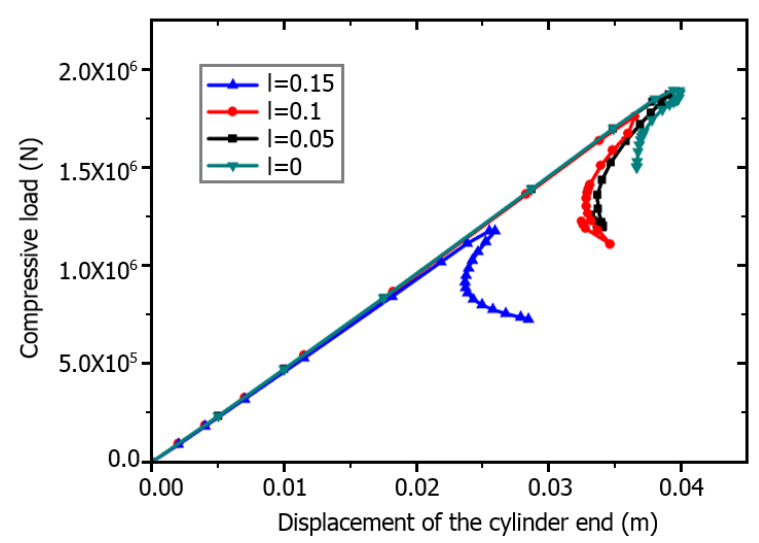

$\mathrm{b}$

Fig. 7 The effect of the laser spot position away from the axis on the CBL (a) The CBL varied with the deviating distance of the laser spot, (b) The compressive load varied with the compressive displacement

\section{Conclusions}

In this study, a three-dimensional thermomechanical coupling finite element model of the CFRP cylindrical shell was developed to simulate the buckling damage process of the cylindrical shell under axial compression and laser irradiation, and the influence of related parameters on the CBL was discussed as well. It can be concluded that the laser irradiation leads to local buckling first and greatly decrease the value of CBL. Additionally, the variation law of CBL with respect to spot size, the shell thickness and the laser spot position was obtained. The value of CBL decreases with the decrease of the shell thickness or the increase of the radius of the spot, and the value of CBL is near quadratically correlated with the shell thickness. Along the cylindrical shell axis direction, the vulnerable location for buckling are two points approaching to the midpoint from two ends instead of the midpoint. Perpendicular to the axis direction, the buckling damage is more likely to happen as the laser deviates from the axis.

\section{Acknowledgements}

This research was funded by Institute of Mechanics, Chinese Academy of Sciences.

\section{References}

1. Lundell, J.; Dickey, R. R. 1979. The response of heatshield materials to intense laser radiation, Proceedings of the AIAA 16th Aerospace Sciences Meeting, USA: Huntsville. https://doi.org/10.2514/6.1978-138.

2. Yi, F.; Liang, J. 2000. Study on the ablation mechanism and models of heatshield composites, Journal of Solid Rocket Technology 23(3): 48-56 (in Chinese). https://doi.org/10.3969/j.issn.1006-2793.2000.04.012.

3. Peng, G.; Du, T.; Liu, F.; Zhang X. 2014. Simulation study of laser energy coupling coefficient in the process of ablating glass fiber / epoxy composites, Chinese Journal of Lasers 41(2): 217-221 (in Chinese). https://doi.org/10.3788/cj1201441.0203001.

4. Park, S. J.; Seo, M.K. 2012. Carbon fiber-reinforced polymer composites: preparation, properties, and applications, Polymer Composites 1: 135-183.

https://doi.org/10.1002/9783527645213.

5. Park, S. J.; Lee, S. Y.; Jin, F. L. 2015. Surface Modification of Carbon Nanotubes for High-Performance Polymer Composites. In Handbook of Polymer Nanocomposites. Processing, Performance and Application, 1st ed.; Editor 1, Kar, K. K., Editor 2, Pandey, J. K., Eds.; Springger-Verlag: Berlin Heidelberg, Germany; Volume B, p. 13-60, ISBN 978-3-642-45228-4. https://doi.org/10.1007/978-3-642-45229-1_34.

6. Kim, M.; Sung, D. H.; Kong, K.; Kim, N.; Kim, B. J.; Park, H. W.; Park, Y. B.; Jung, M.; Lee, S.H.; Kim, S. G. 2016. Characterization of resistive heating and thermoelectric behavior of discontinuous carbon fiberepoxy composites, Composites Part B: Engineering 90: 37-44. https://doi.org/10.1016/j.compositesb.2015.11.037.

7. Xu, H.; Zhang, X.; Liu, D.; Yan, C.; Chen, X.; Hui, D.; Zhu, Y. 2016. Improving interfacial property of carbon fiber/epoxy composites and preserving fiber tensile strength, Composites Part B: Engineering 93: 244-251. https://doi.org/10.1016/j.compositesb.2016.03.033.

8. Zhao, J.; Zhang, G.; Liu X. 1996. A numerical simulation to the deformation of an internally pressured cylinder tank under intensive laser irradiation, Chinese Journal of High Pressure Physics 10(4): 44-49 (in Chinese).

9. Wang, J.; Wang, X.; Wang, F. 2005. Numerical simulations on buckling failure of preloaded cylindrical shell irradiated by high power laser beam, Chinese Journal of High Pressure Physics 19(2): 151-158 (in Chinese).

10. Deng, K. S.; Davies, A. W.; Willams, F. W.; Ji, Z. 2000. Thermo-buckling failure of axially pre-compressed cylindrical shells irradiated by laser beam, AIAA Journal 38(10): 1789-1794. https://doi.org/10.2514/2.849.

11. Bisagni, C.; Cordisco, P. 2006. Post-buckling and collapse experiments of stiffened composite cylindrical shells subjected to axial loading and torque, Composite Structures 73(2): 138-149. https://doi.org/10.1016/j.compstruct.2005.11.055. 
12. Tafreshi, A.; Bailey, C. G. 2007. Instability of imperfect composite cylindrical shells under combined loading, Composite Structures 80(1): 49-64.

https://doi.org/10.1016/j.compstruct.2006.02.031.

13. Frulloni, E.; Kenny, J. M.; Conti, P. 2007. Experimental study and finite element analysis of the elastic instability of composite lattice structures for aeronautic applications, Composite Structures 78(4): 519-528. https://doi.org/10.1016/j.compstruct.2005.11.013.

14. Huang, Y. 2015. Experimental and numerical simulation of multi-field coupling effect of a typical target irradiated by a strong laser. Doctoral Dissertation, Chinese Academy of Sciences University, Beijing (in Chinese).

15. Hu, P.; Chen, F. 2011. Numerical simulation of plane target in airflow under laser irradiation, High Power Laser and Particle Beams 23(7): 1935-1939 (in Chinese). https://doi.org/10.3788/HPLPB20112307.1935.

16. Ma, T.; Xing, X.; Song, H.; Huang, C. 2018. On similarity criteria of thin-walled cylinder subjected to complex thermomechanical loads, Thin-Walled Structures 132: 549-557.

https://doi.org/10.1016/j.tws.2018.09.015.
X. Xing, W. Guo, X. Duan, L. Wang

THERMOMECHANICAL COUPLING DAMAGE AND

ANALYSIS OF BUCKLING LOAD OF LASER

IRRADIATED CFRP CYLINDER SHELL UNDER

AXIAL LOAD

S u m m a r y

This study aims to analyze the effect of thermomechanical coupling damage caused by laser irradiation on a Carbon Fiber Reinforced Polymer (CFRP) cylindrical shell with axial compression using finite element method (FEM). The quasi-static thermomechanical coupling damage process of a typical CFRP structure was studied intensively. First, a thermomechanical coupling model was established, then the influence of the size of the laser spot, the thickness of the shell and the different exposure location of the laser spot on the critical buckling load (CBL) were investigated. The results show that the value of CBL decreases near quadratically as the shell thickness decreases. The value of CBL decreases nonlinearly with increase of the spot size. The CBL decreases and then increases until the laser reaches the midpoint of cylinder axis as the laser moves along the axial direction of the cylinder; the CBL decreases dramatically as the laser deviates from the axis.

Keywords: laser irradiation, axial compression, DFRP cylindrical shell, thermo-mechanical coupling, critical buckling load. 